\title{
Intensity optimization of $x$-ray free-electron laser by using phase shifters
}

\author{
Chi Hyun Shim, Haeryong Yang $®$, Juho Hong, Gyujin Kim, \\ Heung-Sik Kang, and Myung Hoon Cho॰ \\ Pohang Accelerator Laboratory, Pohang University of Science and Technology, \\ Pohang 37673, Korea
}

(Received 6 March 2020; accepted 3 August 2020; published 3 September 2020)

\begin{abstract}
This paper presents a method that uses a gap scan of the phase shifter to optimize the intensity of a freeelectron laser (FEL) by matching its phase with that of the electron beam. Phase shifters are essential instruments, especially for a long undulator line, which is segmented by drift sections. The phase-matched (in-phase) and the $180^{\circ}$ offset (out-of-phase) conditions are investigated using linear theory, FEL simulations, and experiments to understand how the phase shifter affects FEL amplification. We show that the FEL intensity is dominantly reduced by phase mismatch in the saturation region, where the microbunched electron beam is sufficiently developed, and that the difference of FEL intensity between the in-phase and out-of-phase conditions is an effect of evolution of the bunching factor. At the Pohang Accelerator Laboratory x-ray Free-Electron Laser (PAL-XFEL), the gap scan of the phase shifter at $9.7 \mathrm{keV}$ increased FEL intensity by 4 times compared to the calculated gap of the phase shifter. This intensity increase was obtained dominantly in the saturation region.
\end{abstract}

DOI: 10.1103/PhysRevAccelBeams.23.090702

\section{INTRODUCTION}

Most X-ray free-electron laser (XFEL) facilities [1-5] require a long undulator line to obtain sufficient FEL power. Such a line is normally divided into drift sections, each of which includes a quadrupole to focus electron beams, a corrector to control the electron beam path, a beam position monitor, a beam loss monitor, and a phase shifter to match the phase between the electron beam and the x-ray beam [6]. To obtain an optimized FEL intensity, the phase shifters are involved in the interaction of the FEL beam and electron beam, whereas other instruments participate to produce a well-guided electron beam.

Phase matching is an important process to optimize XFEL intensity, because the phase mismatch between the generated x-ray beam and the electron beam can degrade the lasing process. Generally, the phase mismatch is unavoidable, because the x-ray beam travels slightly faster than the electron beam. Though such mismatch can be minimized by adjusting the length of drift section [7], most XFEL facilities utilize phase shifters to compensate actively for the phase mismatch [8-11]. Normally, phase shifters are made up of permanent magnets and act like a compact chicane, so phase shifters elongate the electron

"mh0309@postech.ac.kr

Published by the American Physical Society under the terms of the Creative Commons Attribution 4.0 International license. Further distribution of this work must maintain attribution to the author(s) and the published article's title, journal citation, and DOI. beam path and match the phase of electron beam with the $\mathrm{x}$-ray beam before beams enter the next undulator segment. About the optimal condition of the phase shifter, the Spring-8 Angstrom Compact free-electron Laser (SACLA) reported the changes of the radiation intensity according to the gap of the phase shifter [10], and the European XFEL investigated the spectrum changes by phase mismatch [12]. However, they used only two adjacent undulators to a phase shifter; therefore, the spontaneous undulator radiation is used to find the optimal set of the phase shifter.

Physically, FEL optimization by phase matching can be divided into a linear region and a saturation region. In the linear region, the phase mismatch happens primarily in the drift sections. In the undulator, even though the electron beam is slowed down by the wiggling motion, the phase matching arises self-consistently due to the resonance condition. In the saturation region, the phase matching is quite different due to the microbunching and the undulator tapering. FEL amplification by phase matching in the saturation region can be understood by the so-called "phase jump" phenomenon [13-15]. The key idea is to set the dominant phase of the electron beam to zero, which is the so-called "synchronous phase," before the beam enters the next undulator segment. This adjustment ensures that the electrons can stay in the FEL's amplification region and that the lasing process of FEL can be satisfied.

Finding the phase-matching condition results in the maximum FEL intensity; this condition can be determined by scanning the gap of the phase shifter. The effect of this 
method is clearly seen in the saturation region, where the microbunched electron beam is sufficiently developed and the electrons' phase in phase-energy space is clearly justified. The gap scan of the phase shifter can be conducted to optimize an FEL-lasing condition in various undulator taper configurations with variable-gap undulators [16]. Such a process contributes to a reliable performance which has been realized at the Pohang Accelerator Laboratory x-ray Free-Electron Laser (PAL-XFEL) [17].

In this paper, we use theoretical analysis, simulations, and experiments to quantify how the phase shifter affects the FEL intensity and the FEL gain curve at PAL-XFEL. We compare two representative cases: the phase-matched (in-phase) condition and the $180^{\circ}$ offset (out-of-phase) condition. The study shows that the phase matching in the saturation region is important to optimize FEL intensity, and the accurate phase-matched condition can be realized by the method of the gap scan of the phase shifter.

This paper is organized as follows. Section II presents the gain curve change between two cases of the in- and outof-phase conditions, using 1D linear theory for the linear regime and 1D steady-state FEL simulations with the phase-space analysis for the saturation region. Section III presents the gap scan of the phase shifter with experiments compared to the results of 3D time-dependent simulations. Section IV presents the conclusion.

\section{THEORY AND 1D SIMULATION OF THE IN-PHASE AND THE OUT-OF-PHASE CONDITIONS}

\section{A. Linear regime \\ 1. In-phase condition}

As an electron beam of a given energy propagates an undulator of the magnetic field $B_{u}$ and the undulator period $\lambda_{u}=2 \pi / k_{u}$, where $k_{u}$ is the wave vector of the undulator, radiation is generated with the wavelength $\lambda=2 \pi / k=$ $2 \pi c / \omega$, where $k$ is the wave number, $c \sim 3 \times 10^{8}[\mathrm{~m} / \mathrm{s}]$ is the speed of light in a vacuum, and $\omega$ is the angular frequency. A dominant radiation is satisfied with the resonant condition $\lambda=\lambda_{u}\left(1+0.5 K^{2}\right) /\left(2 \gamma_{0}^{2}\right)$, where $K=93.4 B_{u}[T] \lambda_{u}[m]$ is the undulator parameter and $\gamma_{0}$ is the Lorentz factor of the electron beam. If time-dependent terms are ignored, 1D FEL equations are written as [18]

$$
\begin{aligned}
& \frac{d \theta}{d \hat{z}}=\hat{\eta}, \\
& \frac{d \hat{\eta}}{d \hat{z}}=a e^{i \theta}+a^{*} e^{-i \theta}, \\
& \frac{d a}{d \hat{z}}=-\left\langle e^{-i \theta}\right\rangle_{\text {slice }},
\end{aligned}
$$

where $\theta \equiv\left(k+k_{u}\right) z-\omega t$ is the phase of the electron, $\eta \equiv$ $\left(\gamma-\gamma_{0}\right) / \gamma_{0}$ is the energy-deviation ratio of the electron, and $a$ is the slowly varying envelope of the electric field of radiation. The equations are normalized as follows: $\hat{z}=2 k_{u} \rho z, \hat{\eta}=\eta / \rho$, and $a=e K[\mathrm{JJ}] \mathrm{E} /\left(4 \gamma_{0}^{2} k_{u} m c^{2} \rho^{2}\right)$, where $e$ is the charge of electron, [JJ] is the Bessel harmonic correction factor, $\mathrm{E}$ is the amplitude of the electric field, $m$ is the mass of the electron, and $\rho$ is the FEL parameter. Introducing collective variables to describe the bunching factor $b=\left\langle e^{-i \theta}\right\rangle_{\text {slice }}$ and the collective momentum $P=\left\langle\hat{\eta} e^{-i \theta}\right\rangle_{\text {slice }}$, Eqs. (1) are rewritten by ignoring the nonlinear terms $d P / d \hat{z}=a+a^{*}\left\langle e^{-i 2 \theta}\right\rangle-i\left\langle\hat{\eta}^{2} e^{-i \theta}\right\rangle \approx a$ :

$$
\begin{aligned}
& \frac{d a}{d \hat{z}}=-b, \\
& \frac{d b}{d \hat{z}}=-i P, \\
& \frac{d P}{d \hat{z}}=a .
\end{aligned}
$$

Equations (2) represent the linearized FEL equation, which can be simplified to a third-order differential equation:

$$
\frac{d^{3} a}{d \hat{z}^{3}}=i a
$$

The general solution of Eq. (3) can be given as

$$
\begin{aligned}
a(\hat{z}) & =\sum_{l=1}^{3} C_{l} e^{-i \mu_{l} \hat{z}} \\
& =\frac{1}{3} \sum_{l=1}^{3}\left[a\left(\hat{z}_{0}\right)-i \frac{b\left(\hat{z}_{0}\right)}{\mu_{l}}-i \mu_{l} P\left(\hat{z}_{0}\right)\right] e^{-i \mu_{l} \hat{z}},
\end{aligned}
$$

where $\mu_{l}$ indicates three radiation modes: a simple propagator $\mu_{1}=1$, a damper $\mu_{2}=-(1+i \sqrt{3}) / 2$, and a grower $\mu_{3}=(-1+i \sqrt{3}) / 2$. The initial values are given at $\hat{z}_{0}=0$; $a(0)=C_{1}+C_{2}+C_{3}, b(0)=\mu_{1} C_{1}+\mu_{2} C_{2}+\mu_{3} C_{3}$, and $P(0)=i\left(\mu_{1}^{2} C_{1}+\mu_{2}^{2} C_{2}+\mu_{3}^{2} C_{3}\right)$. Considering a self-amplified spontaneous emission (SASE) case of $a(0)=P(0)=$ 0 with a nonzero initial bunching factor $b(0)=b_{0}$, Eq. (4) simplifies to

$a_{\text {in }}(\hat{z}) \cong \frac{i-\sqrt{3}}{6} b_{0} e^{[(\sqrt{3}+i) / 2] \hat{z}}, \quad\left|a_{\text {in }}(\hat{z})\right|^{2} \cong \frac{1}{9} b_{0}^{2} e^{\sqrt{3} \hat{z}}$,

where only the grower mode is dominant, and note that the one-dimensional gain length is defined as $L_{G}=1 / \sqrt{3}$ from Eq. (5). We also use Eqs. (5) and (2a) to obtain the bunching factor

$$
b_{\text {in }}(\hat{z}) \cong \frac{b_{0}}{3} e^{[(\sqrt{3}+i) / 2] \hat{z}}, \quad\left|b_{\text {in }}(\hat{z})\right|^{2} \cong \frac{1}{9} b_{0}^{2} e^{\sqrt{3} \hat{z}} .
$$




\section{Out-of-phase condition}

Let us consider the out-of-phase condition where the phase is shifted by $\pi$ from the in-phase condition. If we define one phase shifter at the position $\hat{z}=\hat{z}_{0} \neq 0$, then the FEL gain curve follows the in-phase condition until the electron beam meets the phase shifter. After the phase shifter, the electron phase is in the out-of-phase condition, and the FEL gain curve changes. Replacing the electron phase $\theta$ with $\theta+\pi$ yields the linearized FEL equations of Eq. (2) as

$$
\begin{aligned}
& \frac{d a}{d \hat{z}}=b, \\
& \frac{d b}{d \hat{z}}=-i P, \\
& \frac{d P}{d \hat{z}}=-a .
\end{aligned}
$$

Equations (7) can be also reduced to a single third-order differential equation which is the same as Eq. (3). Therefore, the expression of the solution is identical to Eq. (4) except for the initial conditions, which are easily obtained from Eqs. (5), (6), and (2) as

$$
\begin{aligned}
& a\left(\hat{z}_{0}\right)=-\frac{1}{3} b_{0} e^{i\left[\left(\hat{z}_{0} / 2\right)-(\pi / 6)\right]} e^{(\sqrt{3} / 2) \hat{z}_{0}}, \\
& b\left(\hat{z}_{0}\right)=-\left.\frac{d a}{d \hat{z}}\right|_{\hat{z}_{0}}=\frac{1}{3} b_{0} e^{i\left(\hat{z}_{0} / 2\right)} e^{(\sqrt{3} / 2) \hat{z}_{0}}, \\
& P\left(\hat{z}_{0}\right)=\left.i \frac{d b}{d \hat{z}}\right|_{\hat{z}_{0}}=\frac{i}{3} b_{0} e^{i\left[\left(\hat{z}_{0} / 2\right)+(\pi / 6)\right]} e^{(\sqrt{3} / 2) \hat{z}_{0}} .
\end{aligned}
$$

Using Eqs. (8), the solution of the out-of-phase equation is derived as

$$
\begin{gathered}
a_{\text {out }}(z)=\frac{1}{9} b_{0} e^{[(\sqrt{3}+i) / 2] \hat{z}_{0}}\left[(\sqrt{3}+i) e^{-i\left(\hat{z}-\hat{z}_{0}\right)}+i e^{[(-\sqrt{3}+i) / 2]\left(\hat{z}-\hat{z}_{0}\right)}+\frac{\sqrt{3}-i}{2} e^{[(\sqrt{3}+i) / 2]\left(\hat{z}-\hat{z}_{0}\right)}\right], \\
\left|a_{\text {out }}(\hat{z})\right|^{2}=\frac{b_{0}^{2}}{81} e^{\sqrt{3} \hat{z}_{0}}\left[-4 \cos \frac{3\left(\hat{z}-\hat{z}_{0}\right)}{2}\left(e^{(\sqrt{3} / 2)\left(\hat{z}-\hat{z}_{0}\right)}-2 e^{(-\sqrt{3} / 2)\left(\hat{z}-\hat{z}_{0}\right)}\right)+4 e^{-\sqrt{3}\left(\hat{z}-\hat{z}_{0}\right)}+e^{\sqrt{3}\left(\hat{z}-\hat{z}_{0}\right)}\right] .
\end{gathered}
$$

These expressions are available for $\hat{z} \geq \hat{z}_{0}$. Equation (10) agrees well with the 1D static simulation up to saturation at $\hat{z} \lesssim 10$ (Fig. 1).

At $\hat{z}>\hat{z}_{0}$, the FEL gain curve is modulated for a short distance in which the propagator, damper, and grower

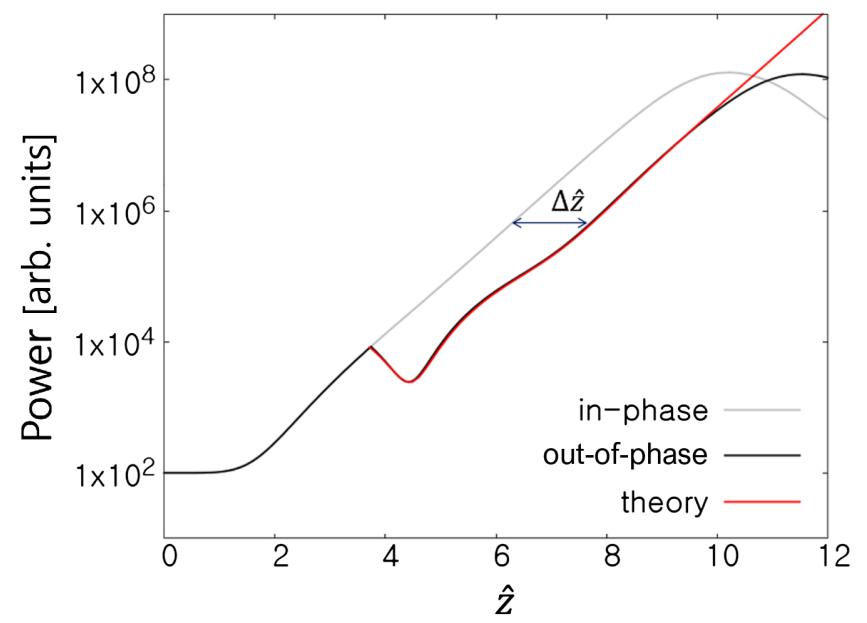

FIG. 1. FEL gain curves of the in-phase and the out-of-phase conditions from the 1D steady state simulation and theory. The phase is shifted by a phase shifter located at $\hat{z}_{0}=3.74$. The shifted distance of the gain curve from the simulation is $\Delta \hat{z}=1.27$, which agrees with the theoretical expectation. modes all participate in the FEL process. However, the grower becomes dominant after the FEL beam propagates farther from the phase shifter, so the result looks like the phase shifter just shifts the gain curve. During the curve modulation, the required length $L_{\text {rec }}$ to recover the initial FEL power is readily obtained by $\left|a\left(\hat{z}_{0}\right)\right|^{2}=\left|a\left(\hat{z}_{0}+L_{\text {rec }}\right)\right|^{2}$, which gives the recovery distance of $L_{\text {rec }} \approx 1.15$ in the normalized unit. Considering the gain length $(1 / \sqrt{3} \approx$ $0.5774)$, the recovery distance is twice the gain length. This recovery distance is constant in normalized units, so it varies according to FEL sets like $k_{u}$ and $\rho$. For example, if $9.7 \mathrm{keV}$ FEL radiation needs $\rho=5 \times 10^{-4}$, $k_{u}=2.42 \times 10^{2}[1 / \mathrm{m}]$ (we used parameters of Table I in Sec. II), the recovery distance is $4.76 \mathrm{~m}$, which addresses that if a undulator segment is longer than $4.76 \mathrm{~m}$, FEL is amplified even in the out-of-phase condition. The distance by which the phase shifter shifts the gain curve may be slightly longer than the recovery distance. To obtain the shifting distance, we further modify Eq. (10). The damping terms of Eq. (10) can be ignored at $\hat{z}>\hat{z}_{0}+L_{\text {rec }}$, so

$$
\begin{aligned}
\left|a_{\text {out }}(\hat{z})\right|^{2} \approx & \frac{b_{0}^{2}}{81} e^{\sqrt{3} \hat{z}_{0}}\left[-4 \cos \frac{3\left(\hat{z}-\hat{z}_{0}\right)}{2} e^{(\sqrt{3} / 2)\left(\hat{z}-\hat{z}_{0}\right)}\right. \\
& \left.+e^{\sqrt{3}\left(\hat{z}-\hat{z}_{0}\right)}\right] .
\end{aligned}
$$


TABLE I. Main parameters measured at PAL-XFEL.

\begin{tabular}{lcc}
\hline \hline Parameter & Value & Unit \\
\hline Electron beam energy & 8.54 & $\mathrm{GeV}$ \\
Bunch charge & 180 & $\mathrm{pC}$ \\
Normalized emittance & 0.4 & $\mu \mathrm{m}$ \\
Peak current & 3 & $\mathrm{kA}$ \\
Undulator parameter $K$ & 1.87 & \\
Undulator period & 26 & $\mathrm{~mm}$ \\
FEL wavelength & 1.28 & $\AA$ \\
Photon energy & 9.7 & $\mathrm{keV}$ \\
\hline \hline
\end{tabular}

Equation (11) is further simplified as

$\left|a_{\text {out }}(\hat{z})\right|^{2}=\frac{b_{0}^{2}}{9} e^{\sqrt{3} \hat{z}}\left[\frac{1}{9}-\frac{4}{9} e^{-(\sqrt{3} / 2)\left(\hat{z}-\hat{z}_{0}\right)}\right] \approx \frac{b_{0}^{2}}{9} e^{\sqrt{3}(\hat{z}-1.27)}$,

where the maximum of the cosine term is selected and the last expression is obtained assuming $\hat{z} \gg \hat{z}_{0}$. Comparing the power expression Eq. (5) of the in-phase condition, the gain length of $\left|a_{\text {out }}(\hat{z})\right|^{2}$ is identical to the in-phase condition $\left|a_{\text {in }}(\hat{z})\right|^{2}$. The only difference is that the required undulator length for the saturation is lengthened by the shifting distance 1.27 (Fig. 1). Consequently, one phase shifter in the out-of-phase condition shifts the gain curve about 1.27 normalized units from the in-phase gain curve.

\section{B. Saturation regime}

As the electron beam enters the saturation region, electrons are bunched locally. A dominant FEL amplification or diminishment is determined by the bunched electrons. According to the Kroll-Morton-Rosenbluth (KMR) model [15], the phase space of the electron and FEL amplification takes place only when the trajectories of the bunched electrons stay in the deceleration region. Setting an appropriate configuration of undulator taper and phase shifters makes as many electrons of the bunched electrons as possible to be positioned in the deceleration region along undulators. To express the electrons' trajectory in phase space, the equations of motion of Eqs. (1a) and (1b) are rewritten as

$$
\frac{d \psi}{d \hat{z}}=\hat{\eta}, \quad \frac{d \hat{\eta}}{d \hat{z}}=-a_{0} \sin \psi,
$$

for which the Hamiltonian is (a)

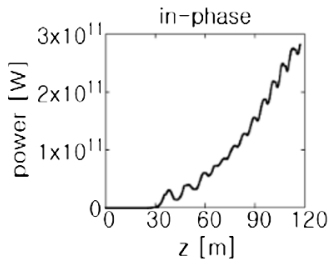

(b)

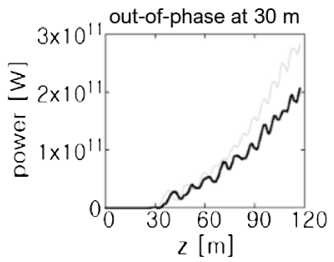

(c)

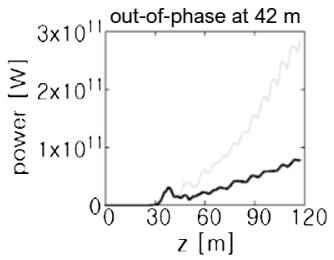

(d)

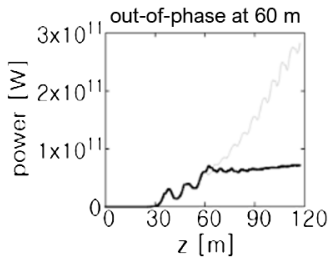

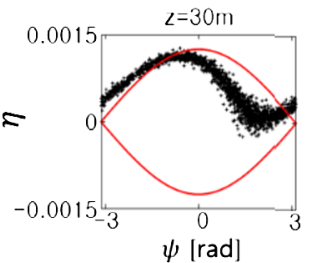
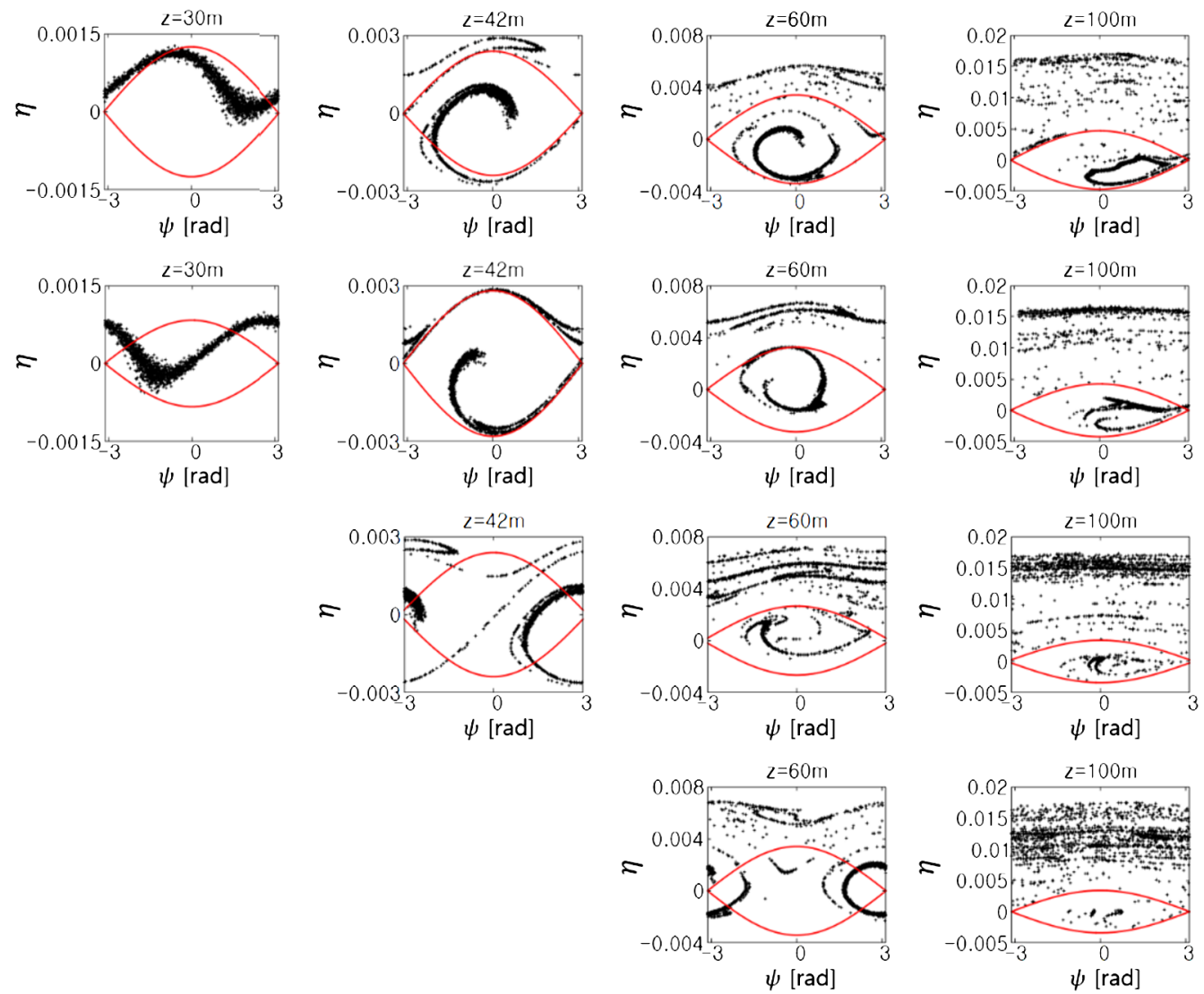

FIG. 2. Comparisons of FEL gain curves with the electron phase distribution from 1D steady state FEL simulation. (a) The in-phase case as the reference. The out-of-phase cases with the phase shifter at (b) 30, (c) 42, and (d) $60 \mathrm{~m}$. Areas between red lines are the phase bucket [Eq. (15)]. 


$$
H=\frac{1}{2} \hat{\eta}^{2}+a_{0}(1-\cos \psi),
$$

where $a_{0} \equiv|a| / 2, \psi \equiv \theta+\phi+\pi / 2$, and $a=|a| e^{i \phi}$. Here, we ignore Eq. (1c), because it is negligibly small in the saturation region compared to the linear region. If we assume that $a_{0}$ is constant, the Hamiltonian is constant, and the separatrix for the trajectory of $H_{\text {sep }}=H(\psi= \pm \pi$, $\hat{\eta}=0)$ can be defined as

$$
\hat{\eta}(\psi)= \pm 2 \sqrt{a_{0}} \cos \frac{\psi}{2} .
$$

When electrons are in the separatrix or so-called "phase bucket," they participate in the FEL process. The undulator taper normally shifts the phase bucket downward, whereas the phase shifter moves the electrons to the right or left in phase space. If the undulator taper is given, the phase shifter dominantly changes the FEL gain curve.

To determine how the positions of the phase shifters affect the FEL gain curve, we conducted a series of 1D static FEL simulations. The FEL gain curve changes most in the out-of-phase condition, so we compared the in-phase and out-of-phase conditions. We assumed 20 undulator modules, each composed of a 5-m-long undulator and a 1 -m-long drift section. The out-of-phase condition is set by simply adding a $\pi$ phase to every electron's phase right after the selected phase-shifter position, which is also located at the entrance of the next undulator segment. To prevent any confusion, we test only one phase shifter for each case; therefore, only the selected module is out of phase and all others are in phase. In this way, we can check how an individual phase shifter affects the FEL gain curve. The simulations considered the in-phase case as a reference [Fig. 2(a)] and the out-of-phase cases where each selected module is located at 30 [Fig. 2(b)], 42 [Fig. 2(c)], and $60 \mathrm{~m}$ [Fig. 2(d)]. The FEL gain curve is reduced in all the out-ofphase conditions, because the phase bucket (red lines) loses electrons. When the selected module is out of phase at $z=30 \mathrm{~m}$ [Fig. 2(b)], the FEL intensity is slightly decreased than the reference case by the lower slope of the FEL gain curve. The highest reduction of FEL intensity occurs when the selected module is out of phase at $z=42 \mathrm{~m}$ [Fig. 2(c)]. After the selected module, the increase rate of the FEL gain curve is quite lowered. In the case of the selected module at $z=60 \mathrm{~m}$ [Fig. 2(d)], the slope of the FEL gain curve after the module position is almost zero, which means that the phase bucket loses electrons significantly after the phase shifter. While the electrons are being bunched as the electron beam propagated further down the undulator modules, the phase bucket can lose electrons easily when the phase shifter is the out-of-phase condition; this observation demonstrates that FEL optimization using phase shifters is very important in the saturation region with microbunching of the electron beam.

\section{3D SIMULATION AND EXPERIMENTS}

\section{A. Gap scan of the phase shifter}

The hard x-ray undulator line at the PAL-XFEL is composed of 20 undulator segments, one self-seeding section, and 19 phase shifters [6] [Fig. 3(a)]; a phase shifter is included in the intersection [Fig. 3(b)]. The delayed distance of electron beam in the intersection is expressed [11] as

$$
s=\frac{1}{2 \gamma^{2}}\left(L_{\mathrm{int}}+\left(\frac{e}{m c}\right)^{2} \cdot P I_{\mathrm{PS}}\right)=n \lambda,
$$

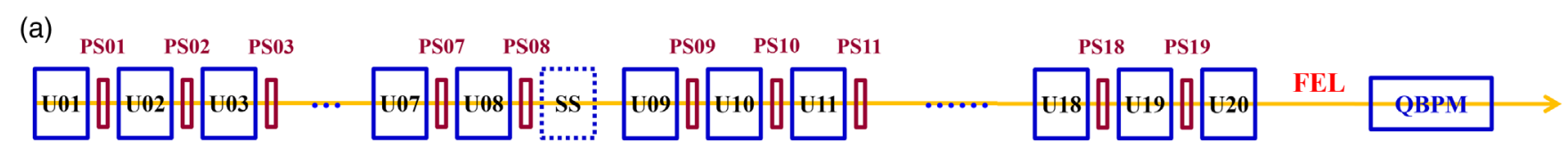

(SS: self-seeding section)
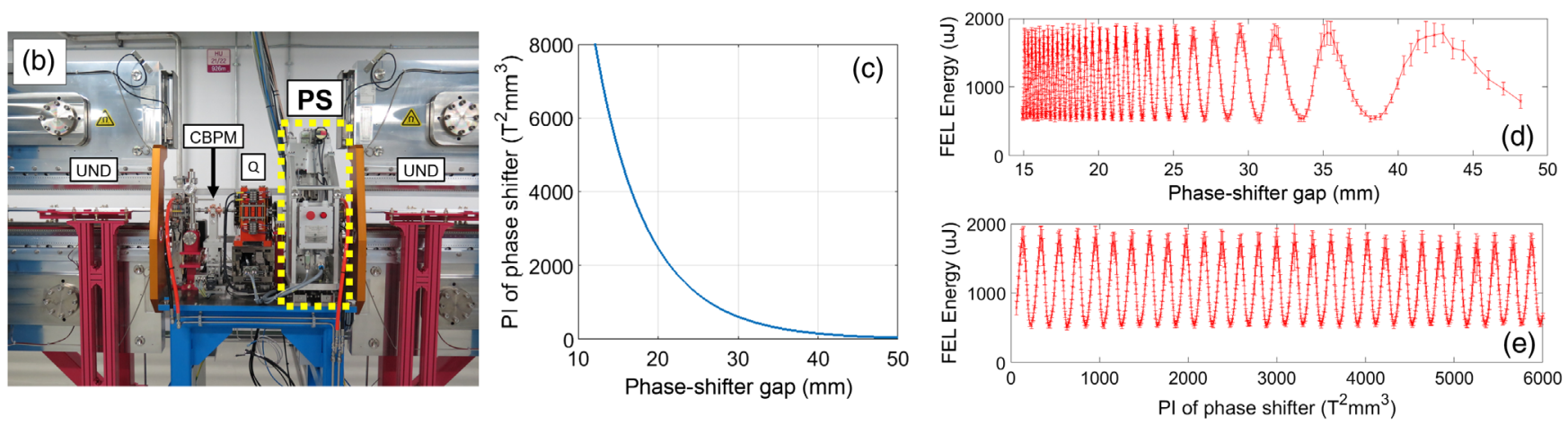

FIG. 3. (a) Schematic layout of the hard x-ray undulator line at PAL-XFEL. (b) Intersection between undulator (UND) modules consisting of cavity beam position monitor (CBPM), quadrupole (Q), and phase shifter (PS). (c) Measured phase integral of the phase shifter as a function of the gap. Measured FEL intensity as a function of (d) the gap of the phase shifter and (e) the phase integral of the phase shifter. 
where $L_{\mathrm{int}}$ is the length of intersection between two undulators and $P I_{\mathrm{PS}}$ is the phase integral of phase shifter. $P I_{\mathrm{PS}}$ can be calculated from the measured magnetic field of phase shifter $B_{y, \mathrm{PS}}$ as

$$
P I_{\mathrm{PS}}=\int_{-\infty}^{\infty}\left(\int_{-\infty}^{z^{\prime \prime}} B_{y, \mathrm{PS}}\left(z^{\prime}\right) d z^{\prime}\right)^{2} d z^{\prime \prime}
$$

A phase-matched condition is normally determined by setting the delayed distance to a multiple of the resonant wavelength. $P I_{\mathrm{PS}}$ can be used as a practical control parameter, because this quantity is easily controlled by changing the gap of the phase shifter. For example, $P I_{\mathrm{PS}}$ increases as the gap of the phase shifter decreases, which is normally operated in the range of $15-50 \mathrm{~mm}$ [Fig. 3(c)]. The optimal gap of the phase shifter is set to where the FEL intensity is at maximum. To measure FEL intensity, PAL-XFEL uses a quadrant beam position monitor (QBPM) located in the optical hutch of the beam line [19]. The QBPM was originally used to diagnose the FEL position, but it can be also used to measure the FEL intensity nondestructively in real time by calibrating its signal to the electron energy loss [20-21]. An example of scanning the gap of the 11th phase shifter (PS11) yields an oscillating response of FEL intensity [Fig. 3(d)]; redrawing by changing the phase-shifter gap to $P I_{\mathrm{PS}}$ clearly shows a periodically oscillating pattern [Fig. 3(e)]. This PI periodicity is simply calculated by plugging the resonance condition into
Eq. (16): $P I_{\mathrm{PS}}=(m c / e)^{2}\left[n \lambda_{u}\left(1+K^{2} / 2\right)-L_{\text {int }}\right]$, so the optimal $P I_{\mathrm{PS}}$ is periodic in $(\mathrm{mc} / e)^{2} \lambda_{u}\left(1+K^{2} / 2\right)$. If $K=1.87$ and $\lambda_{u}=2.6 \mathrm{~cm}$, optimal $P I_{\mathrm{PS}}$ is obtained in every $207.6\left[\mathrm{~T}^{2} \mathrm{~mm}^{3}\right]$ deviation.

The gap of the phase shifter was scanned sequentially from PS01 to PS19. The measured FEL intensity varied as a function of the $P I_{\mathrm{PS}}$, e.g., for PS03, PS09, and PS13. The measured results [Figs. 4(a)-4(c)] agreed well with 3D time-dependent FEL simulation results [Figs. 4(d)-4(f)] using GENESIs1.3 [22]. Major FEL parameters used in the simulation are listed in Table I. In the early stage like PS03, the FEL intensity modulation showed a flat profile around the peak intensity but a steep drop at the minimum intensity. Near the beginning of the saturation regime (PS09), the intensity modulation profile was like a sawtooth. In the postsaturation regime (PS13), the modulation profile was smoothly sinusoidal. Therefore, the intensity profiles does not follow a simple sinusoidal profile but depends on the phase-shifter location; this observation is slightly different from a previous report [10]. For practical reasons, despite these different profiles, a sinusoidal fitting was used to determine the optimal gap of all phase shifters and successfully optimized the FEL. Since the FEL fluctuations by these phase-shifter scans show that the change of the FEL intensity is more sensitive in the saturation region, the optimization of the phase-shifter gap is more effective in the saturation region.
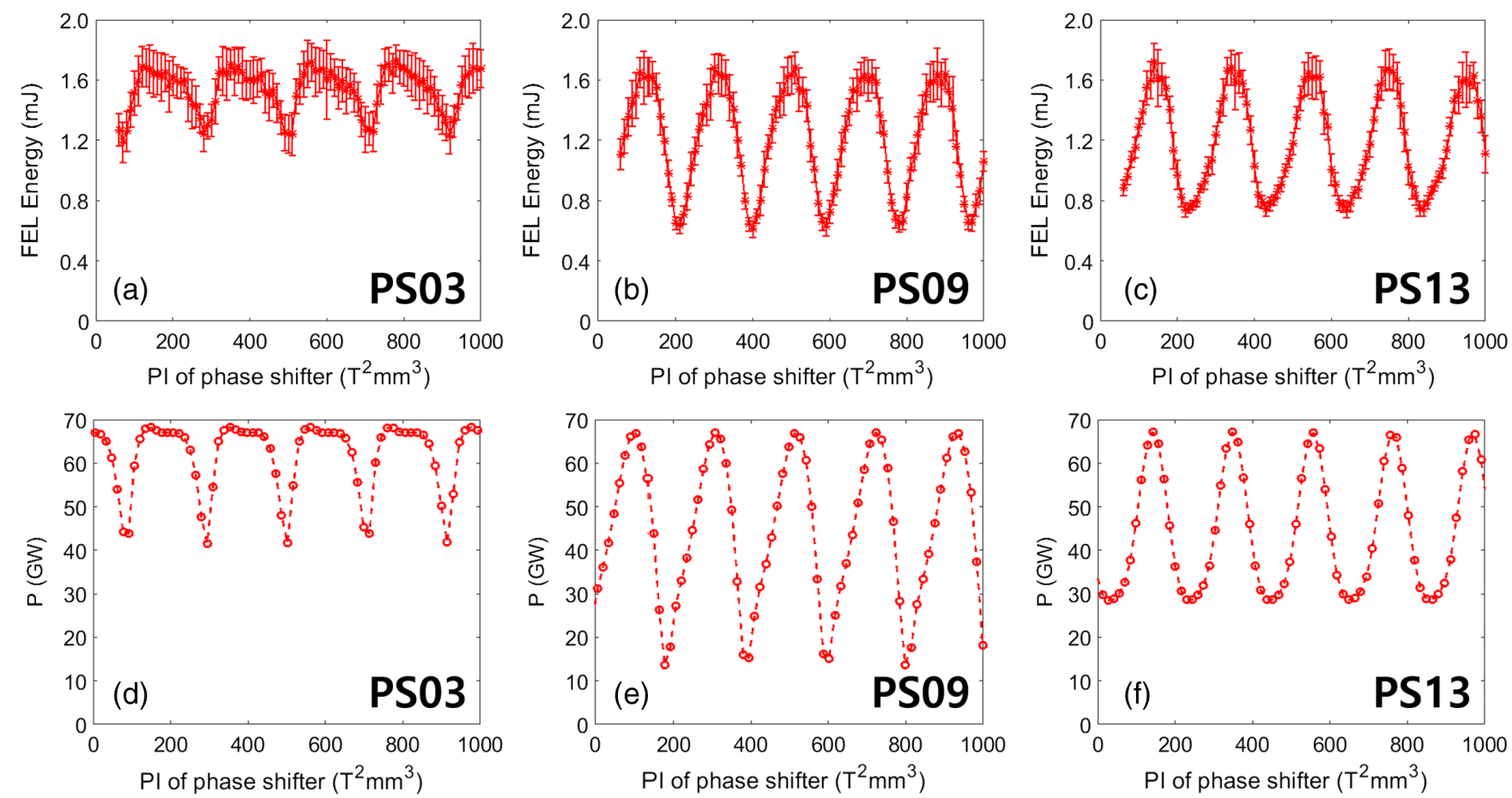

FIG. 4. (a)-(c) Measured FEL intensity at the QBPM (bars, \pm 1 s.d.; $n=100$ ) and (d)-(f) FEL intensity obtained from GENESIS simulation as a function of the phase integral of phase shifter at 18 (PS03), 54 (PS09), and 78 m (PS13). 

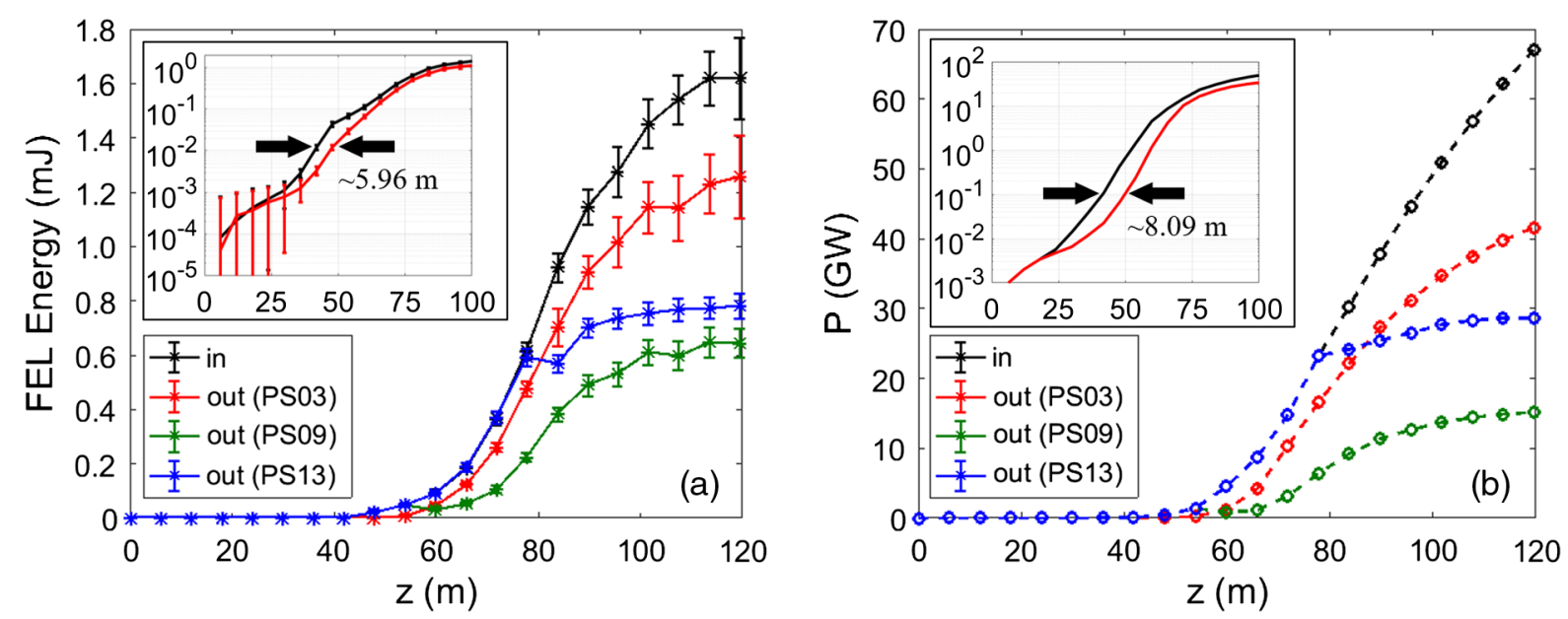

FIG. 5. (a) Measured FEL gain curve from experiment (bars, \pm 1 s.d.; $n=100$ ) and (b) averaged radiation power from simulation along the undulator line for four cases: in phase (black) and out of phase of the phase shifter at 18 (red), 54 (green), and $78 \mathrm{~m}$ (blue). Inset: Log scale of two cases (the in-phase and the out-of-phase PS03).

\section{B. Gain curve and spectrum of the in- and out-of-phase conditions}

The optimal gap of the phase shifter is determined at which FEL intensity is greatest, and it is considered to be the in-phase condition (Fig. 5, black line). To quantify the change in the gain curve at the mismatched phase, one phase shifter was chosen to be set the out-of-phase condition. Measured gain curves [Fig. 5(a)] were compared to the gain curves obtained from GENESIS simulation [Fig. 5(b)]. When the electron beam was set in the outof-phase condition at PS03 (red line) located in the linear region, the gain curve shifted forward from the in-phase gain curve. 1D analysis expected a gain curve shift of $5.26 \mathrm{~m}$, but our experiment and simulation show a longer shift length as shown in the log-scale-plotting insets in Fig. 5. Out of phase at PS09 (green line), where the microbunching of the electron beam is quite progressed, the reduction of the gain curve is dominant. Out of phase at
PS13 (blue line) in the postsaturation regime, the FEL amplification is fully suppressed after the phase shifter.

To check the spectrum effect caused by the phase mismatch, the FEL spectrum at the end of the undulator line was compared through experiments [Fig. 6(a)] and simulation [Fig. 6(b)]. Previous studies reported the spectrum retuning by phase mismatches [7,12], but there is no significant spectrum changes in our study; mostly, intensity drops are observed. Our study assumes FEL amplification by the phase mismatch at single phase shifter; on the other hand, a dominant FEL amplification happens at the resonant wavelength through other undulator segments, and, therefore, the spectrum retuning driven by the phase mismatch at a single phase shifter is invisible.

\section{Bunching factor evolution}

By analyzing the result obtained from the gap scan of the phase shifter, the trend of microbunching evolution
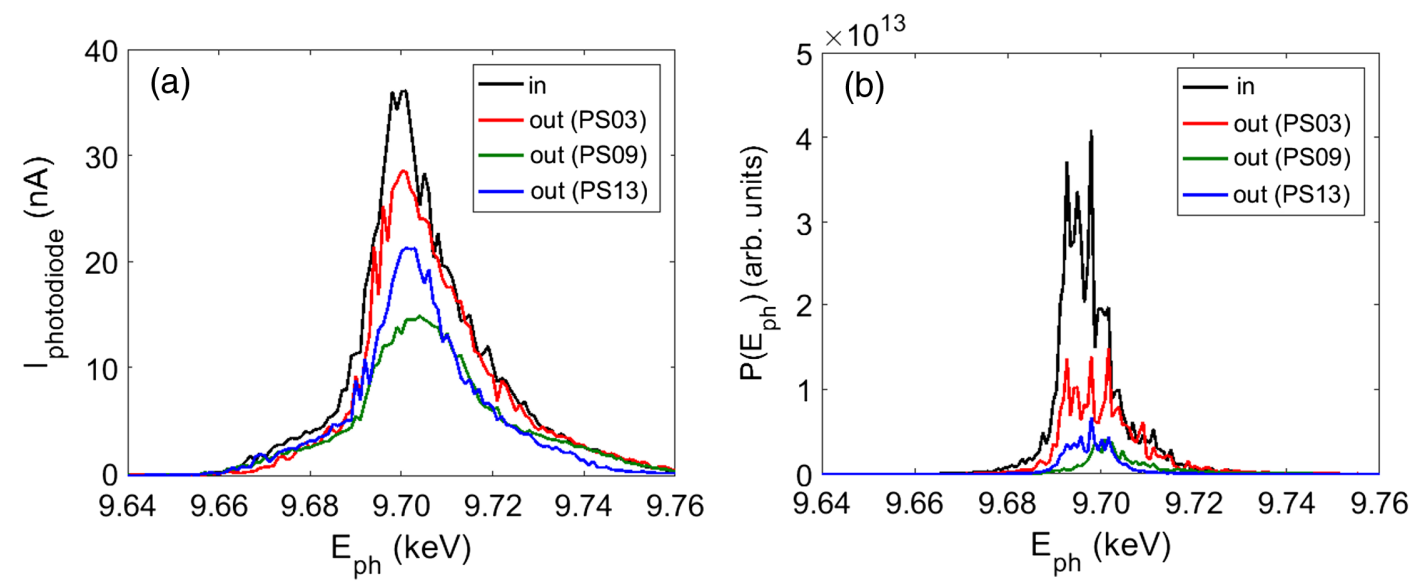

FIG. 6. FEL spectrum from (a) experiment and (b) simulation at the end of the undulator line for four cases: in phase (black) and out of phase of the phase shifter at 18 (red), 54 (green), and $78 \mathrm{~m}$ (blue). 

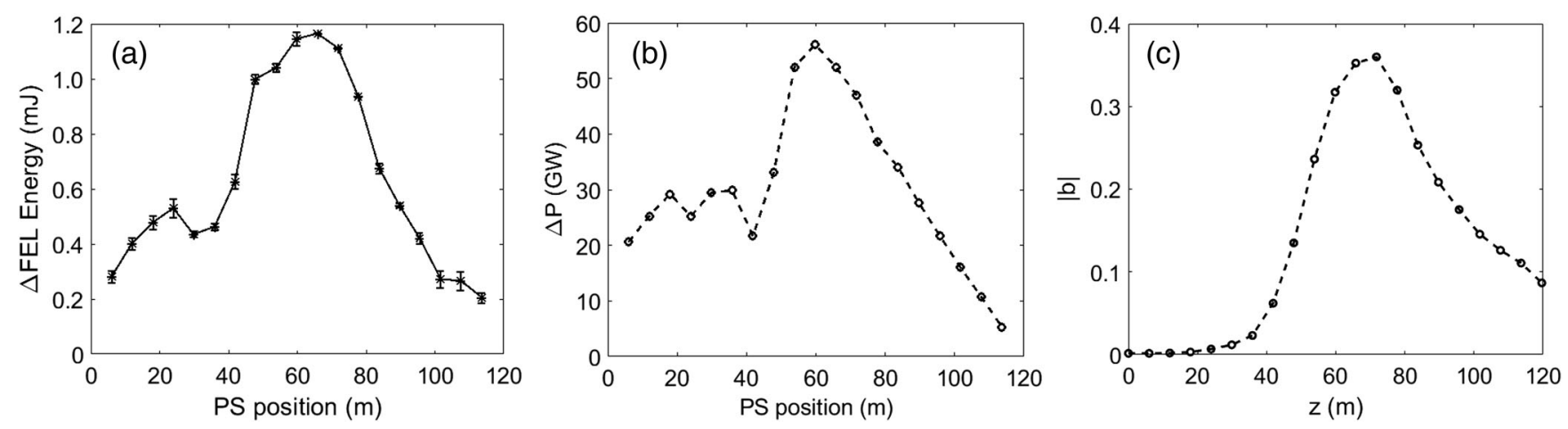

FIG. 7. The difference of maximum and minimum FEL energy from (a) experiment and (b) simulation according to the position of the phase shifter for scanning the gap. (c) Bunching factor along the undulator line obtained from simulation.

can be estimated. The phase-shifter gap scan causes a significant fluctuation in FEL intensity in the saturation region (Fig. 4), so the phase bucket of the out-of-phase condition can lose electrons during the FEL process. Since the fraction of electrons in the phase bucket represents the bunching factor [23] in the saturation region, the trend in the difference between the maximum and minimum of the FEL intensity as a function of phase-shifter position can be considered as the evolution of the bunching factor. In both experiment [Fig. 7(a)] and simulation [Fig. 7(b)], the difference of FEL intensity between the in- and out-ofphase conditions increases as phase-shifter position up to $\sim 60 \mathrm{~m}$ and then decreases. This result concurs with the numerical results [Fig. 7(c)] that the bunching factor is highest in the range of $60 \leq z \leq 70 \mathrm{~m}$, where it overlaps with the position of phase shifters. The overall trend of the difference of FEL intensity between the in-phase and outof-phase conditions is quite similar to the evolution of the bunching factor. Thus, the evolution of the bunching factor can be roughly predicted by scanning the gap of the phase shifter.

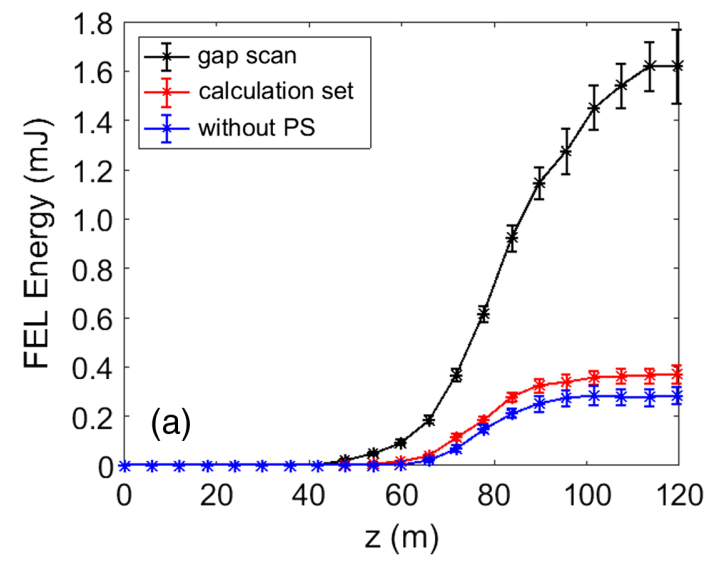

\section{Effect of the phase-shifter gap scan}

Lastly, we compared FEL gain curves in a given undulator taper for three cases to confirm the effect of the phase-shifter gap scan (Fig. 8): (i) the optimized gap case selected by scanning the gap of the phase shifter (gap scan, black line), (ii) the calculated gap case determined by using Eqs. (16) and (17) (calculation set, red line), and (iii) the fully opened gap case $(80 \mathrm{~mm})$ to eliminate the effect of the phase shifter (without PS, blue line). The final FEL energy by the phaseshifter gap scan was $1.62 \mathrm{~mJ}$, but the calculation set gave only $0.37 \mathrm{~mJ}$, and it was decreased to $0.28 \mathrm{~mJ}$ without a phase shifter. The FEL gain curve of the calculation set coincides well with that of the gap scan in the linear region [Fig. 8(b), $z \leq 50 \mathrm{~m}$ ], but the FEL gain curve started to deviate from the beginning of the saturation region. Therefore, setting the phase shifters' gap by calculation is valid only for the linear regime, and it is not appropriate to cover the saturation region where FEL phenomena become highly nonlinear and complex. This result demonstrates that the phase-shifter gap scan is effective to increase the FEL intensity especially for the saturation region.

FIG. 8. Comparisons of the gain curve in (a) linear and (b) log scale; bars, \pm 1 s.d.; $n=100$. The black line is obtained from the phase-shifter gap scans, the red line is setting the phase-shifter gap by calculations using Eq. (16), and the blue line is without the phase-shifter effect. 


\section{SUMMARY}

We studied how the phase shifters affected the FEL amplification in PAL-XFEL. Two representative cases (inphase and out-of-phase conditions) were examined by using theoretical, numerical, and experimental studies. The FEL gain curves differed in the linear and saturation regions. In the linear region, the gain curve in the out-ofphase condition was shifted forward from the in-phase gain curve, but in the saturation region the FEL amplification of the out-of-phase condition was fully suppressed. From the out-of-phase condition of a single phase shifter, only an intensity drop is observed in the FEL spectrum. We also demonstrate that the evolution of the bunching factor can be predicted from the difference of maximum and minimum FEL energy which can be obtained by scanning the gap of the phase shifter. By applying the method of the gap scan of the phase shifter, FEL intensity was increased up to 4 times higher than that from the calculated gap of the phase shifter in our case. Present phase shifters can finely resolve the FEL wavelength and can help to guide optimization of various FEL characteristics such as high harmonics and self-seeding.

\section{ACKNOWLEDGMENTS}

This research was supported by the Ministry of Science and ICT of Korea (MSIT) [Grant No. 2018R1A6B4023605], partly by the Basic Science Research Program [Grants No. 2017R1A2B4007274, No. 2020R1C1C1011840] through the National Research Foundation of Korea (NRF) funded by the MSIT, and by Basic Science Research Program [Grant No. NRF2019R1I1A1A01041573] through the NRF funded by the Ministry of Education.

[1] P. Emma et al., First lasing and operation of an ångstromwavelength free-electron laser, Nat. Photonics 4, 641 (2010).

[2] T. Ishikawa et al., A compact x-ray free-electron laser emitting in the sub-ångström region, Nat. Photonics 6, 540 (2012).

[3] H.-S. Kang et al., Hard x-ray free-electron laser with femtosecond-scale timing jitter, Nat. Photonics 11, 708 (2017).

[4] M. Altarelli, The European x-ray free-electron laser facility in Hamburg, Nucl. Instrum. Methods Phys. Res., Sect. B 269, 2845 (2011).

[5] C. J. Milne et al., SwissFEL: The Swiss X-ray free electron laser, Appl. Sci. 7, 720 (2017).

[6] I. S. Ko et al., Construction and commissioning of PAL-XFEL facility, Appl. Sci. 7, 479 (2017).
[7] H. P. Freund, Phase-matching segmented wigglers in freeelectron lasers, Phys. Rev. E 70, 015501(R) (2004).

[8] R. Ganter et al., Technical overview of SwissFEL undulator line, in Proceedings of the 34th International Free-Electron Laser Conference, Nara, Japan (JACoW, Geneva, Switzerland, 2012), p. 583.

[9] H. G. Lee, D. E. Kim, W. W. Lee, K. H. Park, S. B. Lee, H. S. Suh, Y. G. Jung, and H.S. Kang, Design and fabrication of prototype phase shifter for PAL XFEL, in Proceedings of the 4th International Particle Accelerator Conference, Shanghai, China (JACoW, Geneva, Switzerland, 2013), p. 3564.

[10] T. Tanaka, S. Goto, T. Hara, T. Hatsui, H. Ohashi, K. Togawa, M. Yabashi, and H. Tanaka, Undulator commissioning by characterization of radiation in X-ray free electron lasers, Phys. Rev. Accel. Beams 15, 110701 (2012).

[11] H. H. Lu, Y. Li, and J. Pflueger, The permanent magnet phase shifter for the European X-ray free electron laser, Nucl. Instrum. Methods Phys. Res., Sect. A 605, 399 (2009).

[12] Y. Li and J. Pflueger, Phase matching strategy for the undulator system in the European x-ray free electron laser, Phys. Rev. Accel. Beams 20, 020702 (2017).

[13] A. A. Varfolomeev, T. V. Yarovoi, and P. V. Bousine, Possible enhancement of SASE FEL output field intensity induced by local phase jump, Nucl. Instrum. Methods Phys. Res., Sect. A 407, 296 (1998).

[14] A. Mak, F. Curbis, and S. Werin, Phase jump method for efficiency enhancement in free-electron lasers, Phys. Rev. Accel. Beams 20, 060703 (2017).

[15] N. M. Kroll, P. L. Morton, and M. N. Rosenbluth, Freeelectron lasers with variable parameter wigglers, IEEE J. Quantum Electron. 17, 1436 (1981).

[16] H.-S. Kang and H. Loos, X-ray free electron laser tuning for variable-gap undulators, Phys. Rev. Accel. Beams 22, 060703 (2019).

[17] H.-S. Kang et al., FEL performance achieved at PALXFEL using a three-chicane bunch compression scheme, J. Synchrotron Radiat. 26, 1127 (2019).

[18] Z. Huang and K.-J. Kim, Review of x-ray free-electron laser theory, Phys. Rev. Accel. Beams 10, 034801 (2007).

[19] J. Park, S. Kim, K.-H. Nam, B. Kim, and I. S. Ko, Current status of the CXI beamline at the PAL-XFEL, J. Korean Phys. Soc. 69, 1089 (2016).

[20] K. Tono, T. Kudo, M. Yabashi, T. Tachibana, Y. Feng, D. Fritz, J. Hastings, and T. Ishikawa, Single-shot beamposition monitor for x-ray free electron laser, Rev. Sci. Instrum. 82, 023108 (2011).

[21] M. Kato et al., Pulse energy measurement at the hard x-ray laser in Japan, Appl. Phys. Lett. 101, 023503 (2012).

[22] S. Reiche, GENESIS1.3: A fully 3D time-dependent FEL simulation code, Nucl. Instrum. Methods Phys. Res., Sect. A 429, 243 (1999).

[23] A. Mak, F. Curbis, and S. Werin, Model-based optimization of tapered free-electron lasers, Phys. Rev. Accel. Beams 18, 040702 (2017). 